\title{
Fenologia reprodutiva de Ananas comosus var. erectifolius (L. B. Smith) Coppens \& F. Leal. e sua correlação com a temperatura média do ar e precipitação
}

\section{pluviométrica}

\author{
Reproductive phenology of Ananas comosus var. erectifolius (L. B. Smith) Coppens \& F. Leal. and
} its correlation with average temperature and rainfall

Fenología reproductiva de Ananas comosus var. erectifolius (L. B. Smith) Coppens y F. Leal. y su correlación con la temperatura media del aire y las precipitaciones

\section{Resumo}

A espécie Ananas comosus var. erectifolius, conhecida como curauá, pertence a familia das Bromeliáceas, na região amazônica sua folhagem tem proveito na produção de fibras e também é considerado uma espécie medicinal. Apesar da sua importância, pesquisas sobre o comportamento da fenologia de espécies com grande potencial econômico da Amazônia são escassas. O trabalho teve como objetivo realizar o estudo das fenofases reprodutivas de Ananas comosus var. erectifolius pertencente ao Banco Ativo de Germoplasma da Embrapa Amazônia Oriental correlacionando com a temperatura do ar e a precipitação pluviométrica, com a finalidade de indicar a época mais adequada para a coleta e utilização do material vegetal da espécie. As frequências foram registradas semanalmente de janeiro de 2015 a dezembro de 2019. A atividade fenologica exibiu diferenças entre acessos. O índice de correlação de Spearman apontou uma correlação direta e significativa apenas na frutificação em relação à temperatura. $\mathrm{O}$ registro fenológico mostrou a máxima concentração de grupos de acessos que apresentaram maiores médias de dias de floração em de agosto, setembro e outubro, com destaque em agosto. A frutificação registrou as maiores médias percentuais de dias de frutificação em agosto, setembro, outubro e novembro, com destaque em outubro. Sugere-se a coleta de material vegetal de interesse da espécie de dezembro á julho, cujo registro de número de dias que ocorrem as fenofases sofre reduções. È pertinente executar posteriores estudos de correlação da fenologia com eventos climáticos considerando individualmente o desempenho de cada acesso.

Palavras-chave: Curauá; Fenologia reprodutiva; Precipitação de chuva; Temperatura média do ar.

\begin{abstract}
The species Ananas comosus var. erectifolius, known as curauá, belongs to the family of Bromeliaceae, in the Amazon region its foliage has benefits in the production of fibers and is also considered a medicinal species. Despite its importance, research on the behavior of phenology of species with great economic potential in the Amazon is scarce. The objective of this work was to study the reproductive phenophases of Ananas comosus var. erectifolius belonging to the Active Germplasm Bank of Embrapa Amazônia Oriental correlating with air temperature and rainfall, in order to indicate the most suitable time for the collection and use of plant material of the species. The frequencies were recorded weekly from January 2015 to December 2019. Phenological activity showed differences between accesses. The Spearman correlation index showed a direct and significant correlation only in fruiting in relation to temperature. The phenological record showed the maximum concentration of groups of accessions that had
\end{abstract}


the highest average flowering days in August, September and October, especially in August. Fruiting recorded the highest average percentage of fruiting days in August, September, October and November, especially in October. It is suggested the collection of plant material of interest to the species from December to July, whose registration of the number of days that occur the phenophases undergoes reductions. It is pertinent to carry out further studies on the correlation of phenology with climatic events considering the performance of each access individually.

Keywords: Curaua; Reproductive phenology; Rainfall precipitation; Average air temperature.

\section{Resumen}

La especie Ananas comosus var. erectifolius, conocido como curauá, pertenece a la familia de las Bromeliaceae, en la región amazónica su follaje tiene beneficios en la producción de fibras y también es considerada una especie medicinal. A pesar de su importancia, las investigaciones sobre el comportamiento de la fenología de especies con gran potencial económico en la Amazonía son escasas. El objetivo de este trabajo fue estudiar las fenofases reproductivas de Ananas comosus var. erectifolius perteneciente al Banco de Germoplasma Activo de Embrapa Amazônia Oriental correlacionando con la temperatura del aire y las precipitaciones, a fin de indicar el momento más adecuado para la recolección y uso del material vegetal de la especie. Las frecuencias se registraron semanalmente desde enero de 2015 hasta diciembre de 2019. La actividad fenológica mostró diferencias entre accesos. El índice de correlación de Spearman mostró una correlación directa y significativa solo en fructificación en relación a la temperatura. El registro fenológico mostró la máxima concentración de grupos de accesiones que tuvieron los días de floración promedio más altos en agosto, septiembre y octubre, especialmente en agosto. La fructificación registró el mayor porcentaje de días de fructificación en agosto, septiembre, octubre y noviembre, con un destaque en octubre. Se sugiere la recolección de material vegetal de interés para la especie de diciembre a julio, cuyo registro del número de días que ocurren las fenofases sufre reducciones. Es pertinente realizar más estudios sobre la correlación de la fenología con los eventos climáticos considerando el desempeño de cada acceso de manera individual.

Palabras clave: Curauá; Fenología reproductiva; Precipitación pluvial; Temperatura media del aire.

\section{Introdução}

O curauá assim conhecido porpularmente, pertence à familia das Bromeliáceas, possui o porte herbáceo, comumente encontrado na região amazônica e apresentando duas variedades: roxa e branca, cuja folhagem é aproveitada para a confecçao de fibras no estado do Pará (Lameira et al., 2020).

Segundo Satyanarayana; Guimarães; Wypych, (2007) as fibras oriundas do material botânico são materiais que oforecem vantagens quando comparado aos materiais convencionais utilizados para fabricação de compósitos sinteticos, por serem matérias primas de fontes renováveis, biodegradáveis, exploração de baixo impacto, e que oferece portanto menos risco ao meio ambiente. Segundo Frollini et al., (2000) e Cordeiro et al., (2011) o curauá apresenta fibras longas com boa resistência, maciez e peso reduzido, e por possuir características impulsionaram o seu uso na fabricação de papel, confecção de cordas, barbantes, produtos artesanais, na indústria geotêxtil e automobilística para produção de componentes para bancos e revestimento de automóveis. Estudo feito por Fujihashi e Barbosa (2002) demonstrou que o curauá também é considerado uma espécie medicinal por possuir propriedade antimicrobiana, o que lhe confere maior valor agregado na indústria têxtil. Crestani et al., (2010) afirmam que algumas partes da planta destacan-se como potenciais fármacos.

No Pará esta espécie vem assumindo papel de destaque, não somente por apresentar diversidade de uso e boa adaptabilidade a diferentes condições ambientais, mas também por criar oportunidade de gerar emprego e renda através do incremento de novas tecnologias com benefícios sócio-econômicos e ecológicos (Cordeiro et al., 2011).

Quanto ao seu plantio, segundo Lameira (2009) o curauá é pouco exigente no que se refere á qualidade do solo,porém, é extremamente dependente de sitios agricolas com indice pluviométrico superior a 2.000 mm/ano. Para Souza et, al., (2017) a fenologia do gênero Ananas estão intimamente associado com a cultivar e as condições ambientais (temperatura, umidade, luminosidade). Existe cultivares mais precoces e outras mais tardias, o que influencia no desenvolvimento da planta, florescimento e colheita dos frutos. Seu extrato vegetal possui também potencialidade na adubação orgânica, pois a atividade é contínua e favorece o enriquecimento do solo (Cordeiro et al., 2010).

Apesar da sua importância, pesquisas sobre fenofases de espécies com grande potencial econômico da Amazônia são escassas (Pires et al., 2016). Morellato et al., (2016) afirmam que a fenologia pode ser subdividida em vegetativa (estudo das 
folhas) e reprodutiva (composta pela formação de flores e frutos das plantas). Com o conhecimento da fenologia da espécie, é possível fazer o uso racional dos recursos vegetais, uma vez que será observado qual o estádio adequado para realização de cada operação que envolva o uso da planta, além de ser base para o entendimento dos fatores que estimulam o acontecimento do florescimento e frutificação (Matzenauer, 2007).

Estudos fenológicos permitem indicar padrões dos ciclos vegetativos e reprodutivos bem como suas dependências aos fatores ambientais (Françoso et al., 2014; Vogado et al., 2016).

Desta forma, objetivou-se avaliar as fenofases reprodutivas de Ananas comosus var. erectifolius pertencente ao Banco Ativo Germoplasma da Embrapa Amazônia Orientalcorrelacionando com a temperatura média do ar e a pluviosidade, com a finalidade de indicar a época mais adequada para a coleta e utilização do material vegetal da espécie.

\section{Metodologia}

O estudo foi desenvolvido no Banco Ativo de Germoplasma (BAG) de Curauá pertencente ao horto de plantas medicinais da Embrapa Amazônia Oriental. Foi estudado a fenologia de acessos de Ananas comosus var. erectifolius, todos registrados no Herbário IAN do Laboratório de Botânica da mesma instituição. O nome científico da espécie foi atualizado nas bases de dados da Lista de Espécies da Flora do Brasil (Forzza et al., 2014) e do Mobot Tropicos, do Missouri Botanical Garden (Tropicos, 2014). O material de estudado tem seis procedências de origem, municípios de Alenquer, Santarém, Belém, Bragança, Ponta de Pedras e Monte Alegre (Alelo Vegetal, 2021) todos localizados no Pará- Brasil. Entretanto no local de estudo todos estão submetidos às mesmas condições de cultivo. Este trabalho aborda uma pesquisa de campo, sendo de método quantitativo de acordo com Pereira et al. (2018).

Os dados foram coletados durante cinco dias por semana de janeiro de 2015 a dezembro de 2019. Foram avaliados 42 acessos, através da seleção aleatória de 10 planta/acessos cada indivíduo foi marcado com uma fita. Para o registro das fenofases avaliou-se a floração e frutificação, onde a fase de floração inclui desde o desenvolvimento de botões até o final do período de antese das flores, já a frutificação, desde a formação visível dos frutos até a sua queda segundo Medeiros et al., (2016). A duração das fenofases (floração e frutificação) foi anotada em fichas de campo e posteriormente tabuladas em planilhas, sendo determinadas pelas médias de dias por mês de ocorrência do evento fenológico. Para os fatores climáticos foram utilizados valores médios mensais de temperatura média e pluviosidade (Figura 1) cedidos pela Estação meteorológica da Embrapa Amazônia Oriental Belém- Pa, situada nas coordenadas $1^{\circ} 27^{\prime} 21^{\prime \prime} \mathrm{S}$ de latitude e $48^{\circ} 30^{\prime} 14^{\prime \prime} \mathrm{W}$ de longitude, com altitude de 10m (Figura 1). 
Figura 1. Clima do Local de estudo, temperatura média do ar e precipitação pluviométrica média mensal e anual referente ao período de 2015 a 2019, em Belém-PA.

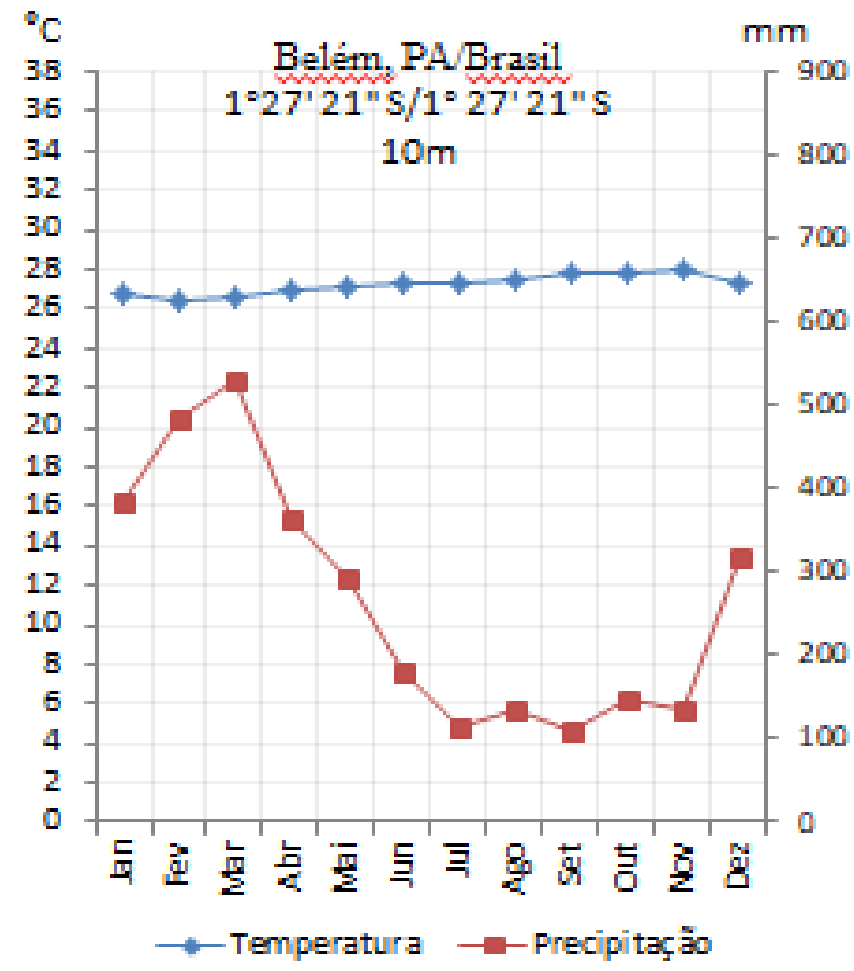

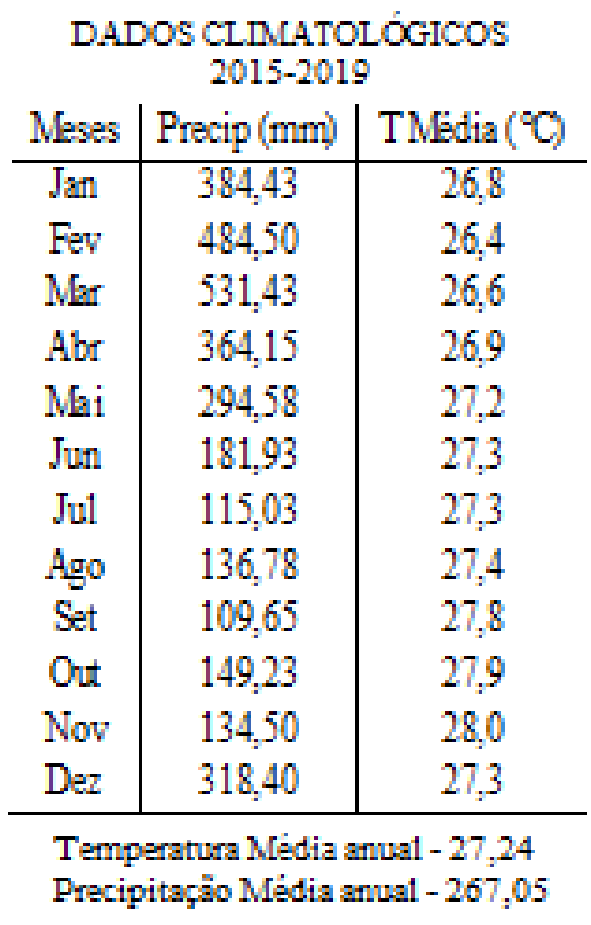

Fonte: Estação Climatológica da Embrapa Amazônia Oriental.

O método adotado para realização das análises foi o índice de atividade sugerido por Bencke \& Morellato (2002), que consiste em registrar a ocorrência ou a ausência do evento reprodutivo em cada espécie. Logo, o uso do termo "pico", refere-se ao pico de atividade, estabelecido por Bencke \& Morellato (2002) como o mês que apresenta o maior número de acessos da espécie em uma determinada fenofase. Para conferir a existência ou não de correlação significativa entre as fenofases e fatores abióticos aplicou-se a correlação de Spearman com o auxilio do software estatístico R (2020), onde o coeficiente de correlação (rho), com sinal negativo (-) indica que as variáveis se correlacionam de maneira inversa, ou seja, o aumento de uma está correlacionado a decréscimo da outra, a intensidade é classificada da seguinte maneira, os valores de rho entre 0,34 e 0,66 indicam relações fracas (rho fraca) e a partir de 0,67 relações fortes (rho forte) como sugeridos por Cann (2002). A elaboração de gráficos e tabelas com o auxílio do Microsoft Excel.

\section{Resultados e Discussão}

No período estudado a pluviosidade variou de 109,65 mm registrada em setembro a 531,43 mm registrada no mês de março. Enquanto a temperatura variou de $26,4^{\circ} \mathrm{C}$ no mês de fevereiro a $28^{\circ} \mathrm{C} \mathrm{em} \mathrm{novembro,} \mathrm{o} \mathrm{índice} \mathrm{de} \mathrm{precipitação} \mathrm{média} \mathrm{e}$ temperatura média anual registrada foram, respectivamente, de $267,05 \mathrm{~mm}$ e $27,24^{\circ} \mathrm{C}$ (Figura 1).

Os acessos de Curauá monitorados revelaram que a espécie, apresenta diferentes padrões fenológicos nos eventos de frutificação e floração, sendo mais acentuado nesta última (Figura 2; Tabela 1). Os ciclos fenológicos de plantas tropicais são complexos, apresentando padrões desiguais de difícil reconhecimento, principalmente em estudos de curto prazo (Bencke \& Morellato, 2002), logo a importância de estudos por anos sequentes.

A floração ocorreu o ano todo, principalmente, nos meses de agosto, setembro e outubro, atingindo o pico de acessos da espécie em agosto com 57,1\% de acessos florando (Figura 2). Estudos com Bromeliaceae em 12 meses de observação 
mostraram quea comunidade de bromélias apresentou floração sequencial em todos os meses no período avaliado (Tagliati1 et al., 2018).

A frutificação ocorreu de forma sequencial o ano todo, esta fenofase foi registrada com maior intensidade de agosto, setembro, outubro e novembro, com pico em outubro (66,7\%). Os períodos do ano em que as fenofases estudadas se mostraram mais intensos assemelharam-se acentuadamente. Logo, onde ocorrem maiores percentuais de acessos expressando floração condiz com os meses em que há tambem elevados números de acessos apresentando frutificações (Figura 2).

Figura 2. Sazonalidade fenológica nos eventos reprodutivos de floração e frutificação do Curauá.

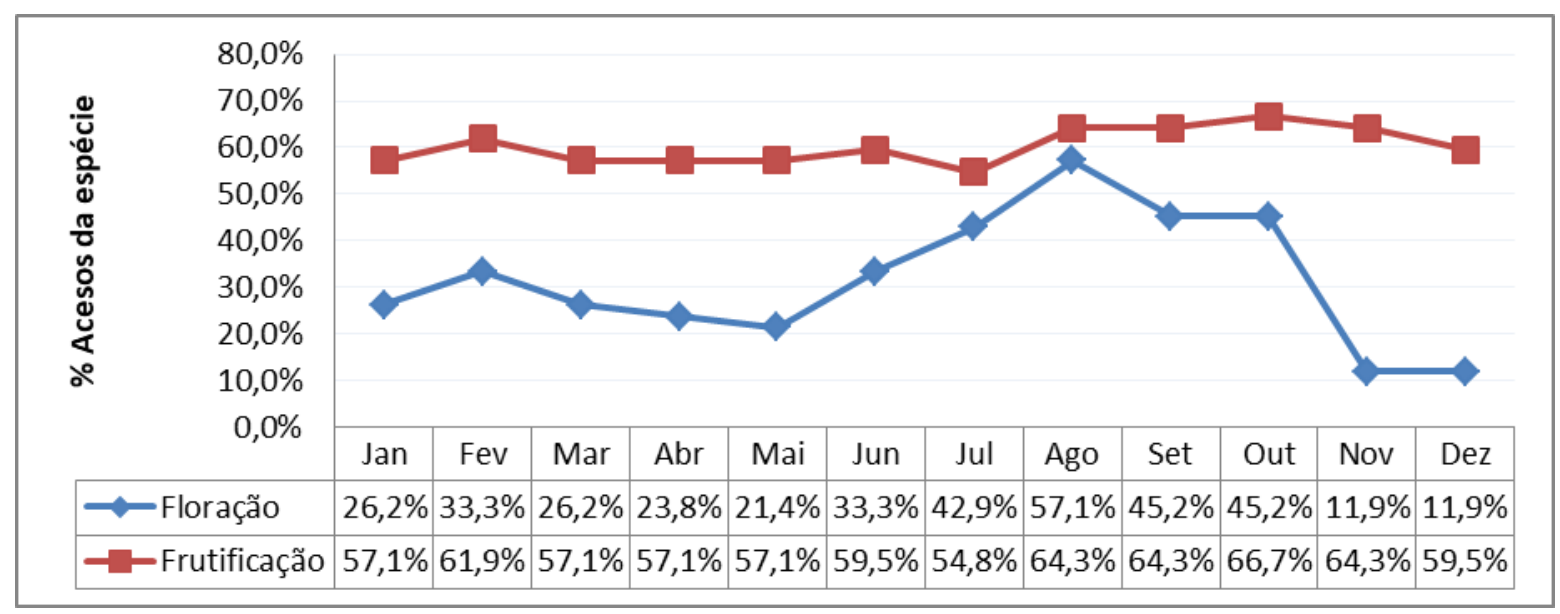

Fonte: Autores.

O índice de atividade (Bencke \& Morellato, 2002), representado na Tabela 1 exibiu o registro de ocorrência ou ausência do evento reprodutivo de 42 acessos cultivados nas mesmas condicões no BAG. Entrertanto, com origem de procedência em seis municípios-PA, observou-se diferenças na atividade fenológica entre os acessos. Como exemplo desta divergência cita-se o acesso AMB 2301(município de Alenquer) não florou e nem frutificou em nenhum mês dos anos, diferente dos demais acessos da mesma procedência. No município de Santarém os acessos AB 101, CB 201, TB 301, DB 501, BFB 601, PAB 901 também houve o registro da ausência dos eventos fenológicos reprodutivos ao decorrer de todo o ano, diferente dos demais acessos, reafirmando desta forma a predominância de baixa sincronia dessa população uma vez que quanto maior o número de indivíduos manifestando a mesma fenofase no mesmo intervalo de tempo, maior é a sincronia dessa população. 
Tabela 1. Atividade fenológica reprodutiva de acessos de Curauá provenientes de seis municípios-Pará, Brasil. $\left(\mathrm{N}^{\circ}=\right.$ número de indivíduos avaliados; floração $=\bullet$; frutificação $=0$ ).

\begin{tabular}{|c|c|c|c|c|c|c|c|c|c|c|c|c|c|}
\hline \multirow{2}{*}{ Municipios } & \multirow{2}{*}{ Acessos } & \multicolumn{12}{|c|}{ Mês } \\
\hline & & Jan & Fev & Mar & Abr & Mai & Jun & Jul & Ago & Set & Out & Nov & Dez \\
\hline \multirow[t]{7}{*}{ Alenquer } & NLR 2200 & 0 & $\bullet \circ$ & $\bullet \circ$ & $\bullet \circ$ & $\bullet \circ$ & $\bullet \circ$ & $\bullet \circ$ & $\bullet \circ$ & $\bullet \circ$ & $\bullet \circ$ & o & $\bullet \circ$ \\
\hline & AMR 2300 & $\circ$ & $\bullet \circ$ & $\circ$ & $\bullet \circ$ & $\bullet \circ$ & $\bullet \circ$ & $\bullet \circ$ & $\bullet \circ$ & $\bullet \circ$ & $\bullet \circ$ & ○ & $\bullet \circ$ \\
\hline & HBR 2400 & $\circ$ & $\bullet \circ$ & $\bullet \circ$ & $\circ$ & ○ & $\bullet \circ$ & $\bullet \circ$ & $\bullet \circ$ & $\circ$ & $\bullet \circ$ & ○ & ○ \\
\hline & SRR 2500 & ○ & $\circ$ & $\bullet \circ$ & $\bullet \circ$ & ○ & $\bullet \circ$ & $\bullet \circ$ & $\bullet \circ$ & $\bullet \circ$ & $\bullet \circ$ & $\circ$ & $\circ$ \\
\hline & FMR 2600 & $\circ$ & $\circ$ & $\bullet \circ$ & $\bullet \circ$ & $\bullet \circ$ & $\bullet \circ$ & $\bullet \circ$ & $\bullet \circ$ & $\bullet \circ$ & $\circ$ & $\circ$ & $\circ$ \\
\hline & AMB 2301 & & & & & & & & & & & & \\
\hline & HBB 2401 & $\bullet \circ$ & $\circ$ & $\bullet \circ$ & $\bullet \circ$ & $\bullet \circ$ & $\bullet \circ$ & $\bullet \circ$ & $\circ$ & $\bullet \circ$ & $\bullet \circ$ & ○ & ○ \\
\hline \multirow[t]{2}{*}{ Belém } & EMBRAPA R 01 & & & & & & $\bullet \circ$ & $\bullet \circ$ & $\bullet \circ$ & $\circ$ & $\bullet \circ$ & $\circ$ & $\circ$ \\
\hline & EMBRAPA B 01 & & & & & & & & & & $\bullet$ & & \\
\hline Bragança & BB 701 & & & & & & & & & & $\bullet \circ$ & & \\
\hline \multirow[t]{9}{*}{ Monte Alegre } & FNR 2100 & $\bullet \circ$ & $\bullet \circ$ & $\bullet \circ$ & $\bullet \circ$ & $\bullet \circ$ & $\bullet \circ$ & $\bullet \circ$ & $\bullet \circ$ & $\bullet \circ$ & $\bullet \circ$ & ○ & ○ \\
\hline & NMB 1301 & & & ○ & $\circ$ & ○ & & & & & & & \\
\hline & JPB1401 & $\bullet \circ$ & $\bullet \circ$ & $\circ$ & $\bullet \circ$ & $\circ$ & $\circ$ & $\circ$ & $\bullet \circ$ & $\bullet \circ$ & $\bullet \circ$ & $\bullet \circ$ & $\circ$ \\
\hline & EFB 1501 & $\bullet$ & $\bullet \circ$ & $\circ$ & $\circ$ & $\circ$ & $\circ$ & & & & & & \\
\hline & JRB 1601 & $\bullet \circ$ & $\bullet \circ$ & $\circ$ & ○ & $\circ$ & $\circ$ & $\circ$ & $\bullet \circ$ & $\bullet \circ$ & $\circ$ & $\circ$ & ○ \\
\hline & SAB 1701 & $\bullet$ & $\bullet \circ$ & ○ & ○ & $\circ$ & $\circ$ & $\bullet \circ$ & $\bullet \circ$ & $\circ$ & $\circ$ & ० & \\
\hline & LAB 1801 & $\circ$ & $\bullet \circ$ & $\circ$ & ○ & $\bullet \circ$ & $\circ$ & $\circ$ & $\circ$ & & & & \\
\hline & NFB 1901 & $\bullet \circ$ & $\bullet \circ$ & ○ & ○ & & & & & $\bullet$ & $\bullet \circ$ & ○ & $\circ$ \\
\hline & ASB 2001 & ○ & $\bullet \circ$ & $\bullet \circ$ & ○ & $\circ$ & $\circ$ & $\bullet$ & $\bullet \circ$ & $\bullet \circ$ & $\bullet \circ$ & $\bullet \circ$ & $\bullet \circ$ \\
\hline \multirow[t]{2}{*}{ Ponta de Pedras } & PPR 400 & $\bullet \circ$ & $\circ$ & $\circ$ & ○ & & $\bullet$ & $\bullet \circ$ & $\bullet \circ$ & $\bullet \circ$ & $\circ$ & $\circ$ & $\circ$ \\
\hline & PPB 401 & & & & & & & & & $\circ$ & $\bullet$ & & \\
\hline \multirow[t]{21}{*}{ Santarém } & MR 001 & $\circ$ & $\circ$ & & & & $\bullet$ & $\bullet \circ$ & $\bullet \circ$ & $\circ$ & $\bullet \circ$ & $\circ$ & ○ \\
\hline & CR 200 & $\circ$ & $\circ$ & ○ & ○ & $\circ$ & $\bullet \circ$ & $\circ$ & $\bullet \circ$ & $\bullet \circ$ & $\bullet \circ$ & $\circ$ & ○ \\
\hline & TR 300 & $\bullet \circ$ & $\bullet \circ$ & $\circ$ & $\circ$ & $\circ$ & $\circ$ & & $\bullet$ & $\bullet$ & $\bullet \circ$ & $\circ$ & $\circ$ \\
\hline & DR 500 & $\circ$ & $\circ$ & $\circ$ & $\bullet \circ$ & $\bullet \circ$ & $\bullet \circ$ & $\bullet \circ$ & $\bullet \circ$ & $\bullet \circ$ & $\bullet \circ$ & $\circ$ & ० \\
\hline & BFR 600 & $\circ$ & $\circ$ & $\bullet$ & $\bullet \circ$ & $\bullet \circ$ & $\circ$ & $\bullet \circ$ & $\bullet \circ$ & $\bullet$ & $\bullet$ & $\bullet \circ$ & $\bullet \circ$ \\
\hline & PEMATEC R01 & $\circ$ & $\circ$ & $\bullet \circ$ & $\bullet \circ$ & $\circ$ & $\bullet \circ$ & $\bullet \circ$ & $\bullet \circ$ & $\bullet \circ$ & $\bullet \circ$ & $\bullet \circ$ & ○ \\
\hline & MB 002 & & & & & & & $\circ$ & & & & & \\
\hline & AB 101 & & & & & & & & & & & & \\
\hline & CB 201 & & & & & & & & & & & & \\
\hline & TB 301 & ० & & & & & & & & & & & \\
\hline & DB 501 & & & & & & & & & & & & \\
\hline & BFB 601 & & & & & & & & & & & & \\
\hline & PEMATEC B02 & & & & & & & & $\bullet$ & $\circ$ & $\circ$ & $\circ$ & \\
\hline & MFR 800 & ○ & $\circ$ & $\circ$ & $\circ$ & $\circ$ & $\bullet \circ$ & $\circ$ & $\bullet \circ$ & $\circ$ & $\circ$ & ० & $\circ$ \\
\hline & PAR 900 & $\circ$ & $\circ$ & & & $\circ$ & ○ & $\bullet \circ$ & $\bullet \circ$ & $\bullet \circ$ & $\circ$ & $\circ$ & $\circ$ \\
\hline & RSR 1100 & $\circ$ & ○ & $\circ$ & $\circ$ & $\bullet \circ$ & ○ & $\bullet \circ$ & $\bullet \circ$ & $\circ$ & $\circ$ & $\circ$ & $\circ$ \\
\hline & ANR 1200 & $\bullet \circ$ & $\bullet \circ$ & $\bullet \circ$ & $\circ$ & $\circ$ & $\circ$ & $\bullet \circ$ & $\bullet \circ$ & $\circ$ & $\circ$ & $\circ$ & $\circ$ \\
\hline & MFB 801 & & & & & & & & $\bullet \circ$ & $\bullet \circ$ & $\circ$ & $\bullet \circ$ & $\bullet \circ$ \\
\hline & РАB 901 & & & & & & & & & & & & \\
\hline & RSB 1101 & & & & & & & & $\circ$ & $\bullet \circ$ & ○ & $\circ$ & $\circ$ \\
\hline & ANB 1201 & $\bullet$ & $\bullet \circ$ & $\circ$ & & ○ & ○ & & $\circ$ & & & & \\
\hline
\end{tabular}


Para a correlação de Spermam (Tabela 2) considerou-se a espécie em geral e o agrupamento dos 42 acessos em seis grupos os quais consideraram a procedência do material (seis municípios), muito embora todos os acessos estivessem submetidos às mesmas condições climáticas e de cultivo no BAG de Curauá. De maneira geral, a espécie apresentou correlação direta e significativa apenas na fenofase de frutificação com a temperatura $(P=0.0218)$, sendo esta classificada como fraca (rho fraca, entre $>0,34$ e $<0,66$ ) com coeficiente de correlação (rho) $=0.651123$. A intensidade de frutificação de espécies nativas, plantadas em núcleos de diversidade pode estar relacionada a fatores abióticos, já que não houve correlação com as variáveis ambientais, Silva (2019). Para Machado (2018) espécies do componente arbustivo-arbóreo a temperatura foi a variável abiótica que mais se correlacionou de forma direta com as fenofases, principalmente a frutificação indicando que fatores climáticos exercem influência sobre tal atividade fenológica.

Para a floração houve identificação de correção não significativa $(\mathrm{P}>0,05)$ entre as variáveis climáticas, cujos valores obtidos foram para precipitação rho $=-0.428828$ e $\mathrm{P} 0.1642$ e para temperatura rho $=0.251327$ e $\mathrm{P}=0.43070$. Para a correlação fenofases reprodutivas x precipitação, ambas apresentam coefiente de correlaçao de Spermam negativos (-) o que permite elucidar uma relação inversa, logo quanto maior o índice pluviométrico a evento de flores e frutos tende a ser menor (Tabela 2. Figura 3A e 5A).

Houve também correlações significativas forte, fraca e correlações não significativas entre os acessos. Quando se observou os distintos grupos, em Alenquer, Bragança e Ponta de Pedras as fenofases não se relacionaram com nenhum fator abiótico de forma significativa. Diferente dos grupos procedentes de Belém, Monte Alegre e Santarém nos quais a frutificação para os dois fatores climáticos teve correlação significativa algumas mais expressivas outras menos, rho forte $>0,67=\left({ }^{2}\right)$; rho fraca entre $>0,34$ e $<0,66=\left({ }^{1}\right)$, a presença de floração se correlacionou de forma significativa com o fator precipitação apenas no grupo de acessos com origem de Santarém.

Tabela 2. Correlações de Spearman (rho) entre a média mensal das variáveis climáticas e os eventos fenológicos de acessos de Curauá com origem de seis municípios, Pará- BR.

\begin{tabular}{|c|c|c|c|c|c|}
\hline \multirow{2}{*}{$\begin{array}{l}\text { Procedência } \\
\text { (grupos) }\end{array}$} & \multirow{2}{*}{ Fatores abióticos } & \multicolumn{2}{|l|}{ Floração } & \multicolumn{2}{|l|}{ Frutificação } \\
\hline & & rho & $\mathbf{P}$ & rho & $\mathbf{P}$ \\
\hline \multirow[t]{2}{*}{ Alenquer } & Temperatura & $0.106284 \mathrm{~ns}$ & 0.7423 & $0,00000 \mathrm{~ns}$ & 0,0000 \\
\hline & Precipitação & $-0.32389 \mathrm{~ns}$ & 0.3044 & $0,00000 \mathrm{~ns}$ & 0,0000 \\
\hline \multirow[t]{2}{*}{ Belém } & Temperatura & $0.448589 \mathrm{~ns}$ & 0.1435 & $0.86294 * 2$ & 0.0003 \\
\hline & Precipitação & $-0.44123 \mathrm{~ns}$ & 0.151 & $-0.80792 * 2$ & 0.0014 \\
\hline \multirow[t]{2}{*}{ Bragança } & Temperatura & $0.395819 \mathrm{~ns}$ & 0.2028 & $0.39581 \mathrm{~ns}$ & 0.2028 \\
\hline & Precipitação & $-0.13101 \mathrm{~ns}$ & 0.6848 & $-0.1310 \mathrm{~ns}$ & 0.6848 \\
\hline \multirow[t]{2}{*}{ Monte Alegre } & Temperatura & $-0.14389 \mathrm{~ns}$ & 0.6555 & $-0.5601 * 1$ & 0.0581 \\
\hline & Precipitação & $-0.02143 \mathrm{~ns}$ & 0.9473 & $0.67749 * 2$ & 0.0154 \\
\hline \multirow[t]{2}{*}{ Ponta de Pedra } & Temperatura & $0.388973 \mathrm{~ns}$ & 0.2114 & $0.5057 \mathrm{~ns}$ & 0.5057 \\
\hline & Precipitação & $-0.53108 \mathrm{~ns}$ & 0.07561 & $-0.2531 \mathrm{~ns}$ & 0.4272 \\
\hline \multirow[t]{2}{*}{ Santarém } & Temperatura & $0.484873 \mathrm{~ns}$ & 0.1101 & $0.77554 * 2$ & 0.0030 \\
\hline & Precipitação & $-0.694213 *$ & 0.01225 & $-0.58657 *$ & 0.0449 \\
\hline \multirow[t]{2}{*}{ Espécie } & Temperatura & $0.251327 \mathrm{~ns}$ & 0.43070 & $0.651123 * 1$ & 0.0218 \\
\hline & Precipitação & $-0.428828 \mathrm{~ns}$ & 0.1642 & $-0.38074 \mathrm{~ns}$ & 0.2221 \\
\hline
\end{tabular}

rho $=$ coeficiente de correlação de Spearman

$* \mathrm{P}<0,05=$ Valores significativos segundo o teste de correlação de Spearman

rho forte $>0,67=\left({ }^{2}\right)$; rho fraca entre $>0,34$ e $<0,66=\left({ }^{1}\right)$.

Fonte: Autores. 
A espécie Ananas comosus apresentou variação no padrão de acontecimento de dias de frutificação e floração entre os grupos de acessos. O registro fenológico mostrou a máxima concentração de grupos de acessos que apresentaram maiores médias de dias de ocorrência de floração em agosto, setembro e outubro, com destaque em agosto com valor médio de $17 \%$ (Alenquer) para os dias analisados. Esses valores coincidem com os menores valores médios de pluviosidade (Figura 3A). Os meses que apresentaram as menores frequências de dias de floração foram novembro e dezembro coincidindo com acentuados registros de temperatura (Figura 3B). A atividade reprodutiva de floração de vegetação arbustivo-arbóreas segundo Machado (2018) foi mais concentrada em determinado período, com intensidade da floração na estação seca (elevadas temperaturas e baixa precipitação).

Figura 3. Percentual de dias por mês de ocorrência do evento fenológico de floração de Curauá e Precipitação Pluviométrica (A) e Temperatura Média do ar (B), no período de janeiro de 2015 a dezembro de 2019.
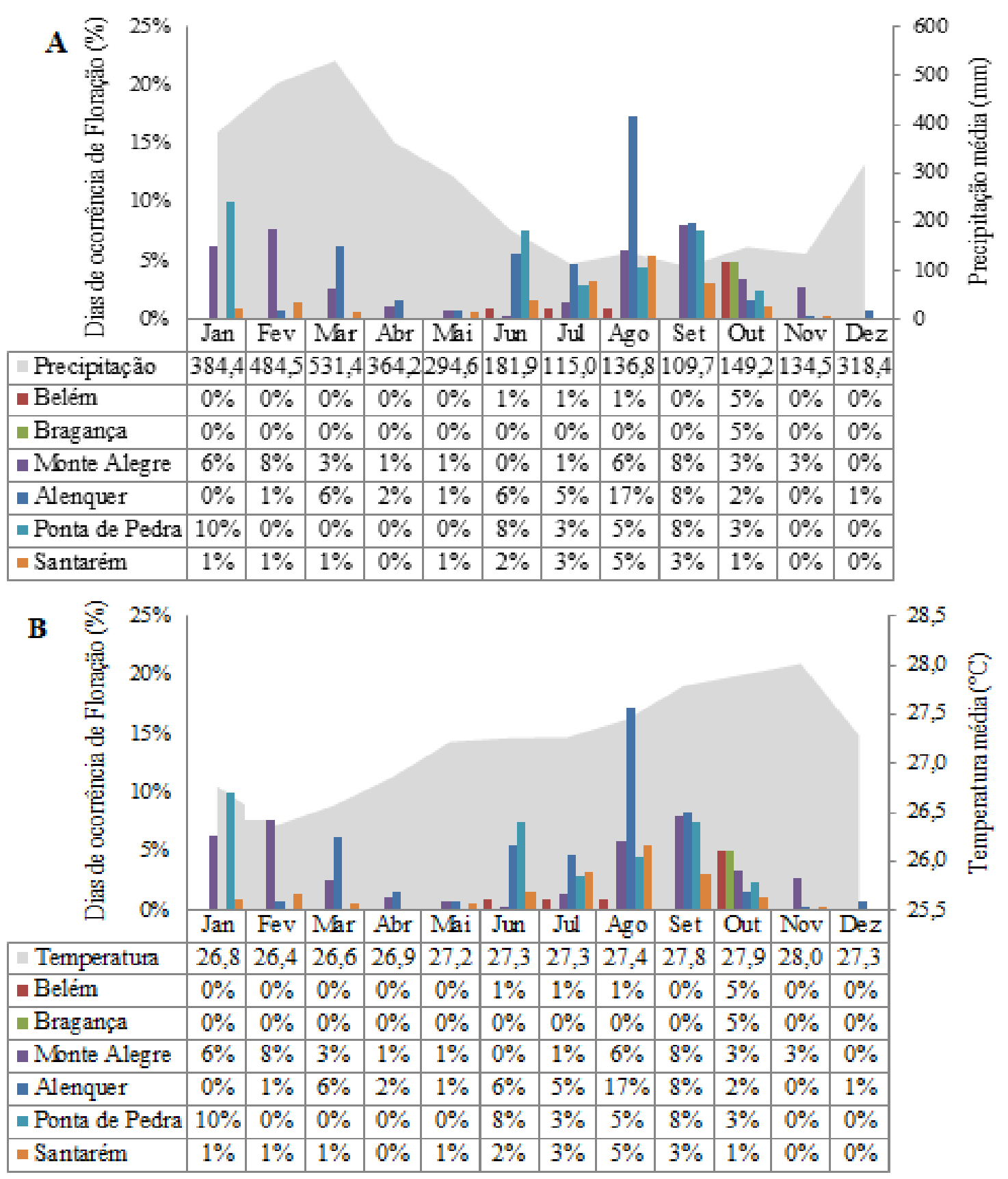

Fonte: Autores. 
O evento fenológico de frutificação registrou as maiores médias percentuais de dias de frutificação para os meses de agosto setembro, outubro e novembro, com destaque em outubro com valor médio de $41 \%$ (Alenquer) para os dias observados, coincidindo com valores baixos de precipitação. Uma redução na frequência de dias desta fenofase nos grupos de acessos avaliados foi registrada de dezembro á julho, coincidindo com períodos de menores médios de temperatura do ar (Figuras 4A e 4B). Resultados semelhantes foram encontrado por Campelo et al. (2017) em estudo com o acesso de curauá Diamantina que apresentou floração e frutificação com maiores médias de número de dias registradas em agosto período de menores valores de precipitação pluviométrica e início de elevada temperatura media do ar.

Figura 4. Percentual de dias por mês de ocorrência do evento fenológico de frutificação de Curauá e Precipitação Pluviométrica (A) e Temperatura Média do ar (B), no período de janeiro de 2015 a dezembro de 2019.

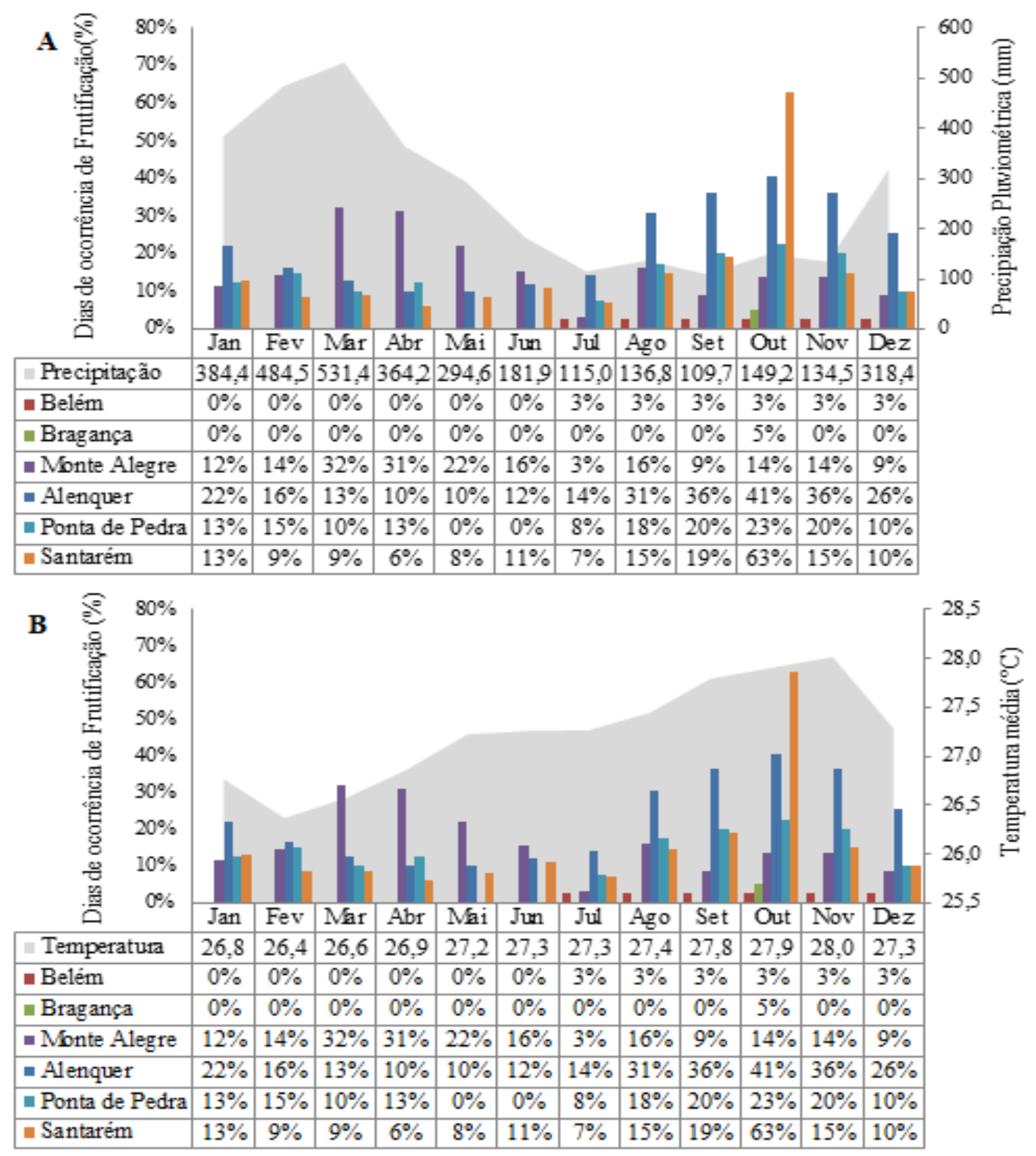

Fonte: Autores.

Assim como para a espécie em estudo, outras espécies vegetais tendem alguma relação com fatores abioticos como temperatura e precipitação (Figuras 3 e 4), mesmo que em alguns períodos ou para determinados acessos, estatisticamente as 
correlações se classifiquem como não significativas. A fenologia da espécie frutifera Psidium cattleianum respondeu diretamente à oscilação de temperatura e precipitação. Após um curto período de seca e um acréscimo gradual da temperatura, no final de julho, começaram os brotos, seguidos de seu crescimento até novembro. Entre agosto e novembro, houve presença de botões florais de Psidium cattleianum, coincidindo com o período de maiores temperaturas e aumento da regularidade da precipitação (Neto et al., 2020).

\section{Conclusão}

O estudo fenológico da espécie Ananas comosus evidenciou variações proeminente para fenologia reprodutiva, com atividade fenológica distintas entre os acessos, além de correlações com significância, intensidade e proporções desiguais para as variáveis analisadas nos grupos de acessos observados. A fase de frutificação da espécie esta diretamente correlacionada de modo considerável ao fator temperatura média do ar.

Contudo sugere-se a coleta de material vegetal de interesse da espécie nos meses de dezembro á julho, cujo registro de número de dias que ocorrem as fenofases sofre reduções. È pertinente executar trabalhos posteriores que abranjam o estudo de correlação da fenologia com eventos climáticos avaliando o comportamento individual de cada acesso.

\section{Agradecimentos}

Os autores agradecem à Coordenação de Aperfeiçoamento de Pessoal de Nível Superior (CAPES) pelo apoio e bolsa de estudo de Doutorado, a Embrapa Amazônia Oriental por ceder o local e a estrutura e ao Programa de Doutorado da Rede Bionorte - Biodiversidade e Biotecnologia.

\section{Referências}

Bencke, C. S., \& Morellato, L. P. C. (2002). Comparação de dois métodos de avaliação da fenologia de plantas, sua interpretação e representação. Brazilian Journal of Botany, 25(3), 269-275.

Bergamaschi, H. (2007). O clima como fator determinante da fenologia das plantas. Fenologia: ferramenta para conservação, melhoramento e manejo de recursos vegetais arbóreos. Colombo: Embrapa Florestas, 1, 291-310.

Campelo, M., Lameira, O., Mavão, L. D. S., \& de Assis, R. M. A. (2017). Fenológia da espécie-Ananas comosus (L.) Merr. var. erectifolius (LB Smith) Coppens \& F. Leal. In Embrapa Amazônia Oriental-Artigo em anais de congresso (ALICE). In: Seminário De Iniciação Científica Da Embrapa Amazônia Oriental, 21., 2017, Belém, PA. Anais. Belém, PA: Embrapa Amazônia Oriental, 2017.

Cann, A. J. (2013). Maths from scratch for biologists. John Wiley \& Sons.

Cordeiro, I. M., Lameira, O. A., de Barros, P. L., \& Malheiros, M. A. D. M. (2010). Comportamento do curauá sob diferentes níveis de radiação fotossinteticamente ativa em condições de cultivo. Revista Brasileira de Ciências Agrárias, 5(1), 49-53.

Cordeiro, I., Andrade, A. D. S., Ferreira, G., \& Braga, R. D. S. (2011). Cultivo e produção agroambiental sustentável de curauá (Ananas comosus var. erectifolius (LB Sm.) Coppens \& F. Leal, Bromeliaceae) no Nordeste paraense-PA. In Embrapa Amazônia Oriental-Artigo em anais de congresso (ALICE). In: CONGRESSO NACIONAL DE MEIO AMBIENTE DE POÇOS DE CALDAS 8., 2011, Poços de Caldas. Anais... Poços de Caldas: GSC.

Crestani, M., Barbieri, R. L., Hawerroth, F. J., Carvalho, F. I. F. D., \& Oliveira, A. C. D. (2010). Das Américas para o mundo: Origem, domesticação e dispersão do abacaxizeiro. Ciência Rural, 40(6), 1473-1483.

da Silva, M. P. K. L., Rovedder, A. P. M., Hummel, R. B., Piaia, B. B., Toso, L. D., Felker, R. M., \& Matiello, J. (2019). Desenvolvimento inicial e fenologia em núcleos de restauração no bioma Mata Atlântica, Sul do Brasil1. Revista Brasileira de Ciências Agrárias (Agrária), 14(1), 5612.

Embrapa. (2021). Embrapa Recursos Genéticos E Biotecnologia. Núcleo de Tecnologia da Informação (NTI). Alelo Vegetal.

Forzza, R. C., Costa, A., Walter, B. M. T., Pirani, J. R., Morim, M. P., Queiroz, L. P. , Martinelli, G., Peixoto, A. L., Coelho, M. A. N., Baumgratz, J. F. A., Stehmann, J. R., \& Lohmann, L. G. Angiospermas in: . Jardim Botânico do Rio de Janeiro. 2014. http://reflora.jbrj.gov.br/jabot/floradobrasil/FB128482.

Françoso, R., Guaraldo, A. D. C., Prada, M., Paiva, A. O., Mota, E. H., \& Pinto, J. R. R. (2014). Fenologia e produção de frutos de Caryocar brasiliense Cambess. e Enterolobium gummiferum (Mart.) JF Macbr. em diferentes regimes de queima. Revista Árvore, 38(4), 579-590.

Frollini, E., Leão, A. L. \&Mattoso, L. H. S. (2000). Natural polymers and agrofibers composites. pg. 257-272, Botucatu, Brasil, USP e UNESP.

Fujihashi, G. D. A., \& Barbosa, W. L. R. (2002). Ananas erectifolius (Curauá): padronização dos extratos, frações e do material vegetal. Revista Científica da 
UFPA, 3, 1-6.

Köppen, W. (1948). Climatologia. Fondo de Cultura Económica.

Kosera Neto, C., Radaelli, J. C., Guollo, K., Wagner Júnior, A., Zanela, J., \& Frazon, R. C. (2020). Acompanhamento fenológico de araçazeiros na Região Sudoeste do Paraná. Research, Society and Development, 9(10), e8319109180. 10.33448/rsd-v9i10.9180.

Lameira, O. A. (2009). Aspectos técnicos e sócio-econômicos da aplicação da micropropagação na produção de fibras: o caso do curauá. In Embrapa Amazônia Oriental-Artigo em anais de congresso (ALICE). In: Congresso Brasileiro De Floricultura E Plantas Ornamentais, 17. , Congresso Brasileiro De Cultura De Tecidos De Plantas, 4., 2009, Aracaju. Ciência, inovação e sustentabilidade. Aracaju: Embrapa Tabuleiros Costeiros , Cruz das Almas: Embrapa Mandioca e Fruticultura Tropical.

Lameira, O. A., Cordeiro, I. M. C. C., \& Pires, H. C. G. (2020). Avaliação dos descritores morfoagronômico e morfoanatomia da lâmina foliar de Pilocarpus microphyllus Stapf ex Wardleworth, Rutaceae, Ananas comosus var.erectifolius(LB Smith)Coppens\&F.Leal,Bromeliacea e Psychotria ipecacuanha(Brot.) Stokes,Rubiaceae. Appris.

Machado, W. D. J. (2018). Fenologia da vegetação em áreas de Areias Brancas no Parque Nacional Serra de Itabaiana, Sergipe, Brasil.

Medeiros, A., Rocha, T. T., Germano, C. M., de Assis, R. M. A., \& Lameira, O. (2016). Fenologia reprodutiva de Quassia amara L.(Simaroubaceae). Embrapa Amazônia Oriental-Artigo em periódico indexado (ALICE).

Morellato, L. P. C., Alberton, B., Alvarado, S. T., Borges, B., Buisson, E., Camargo, M. G. G., \& Peres, C. A. (2016). Linking plant phenology to conservation biology. Biological Conservation, 195, 60-72.

Peel, M. C., Finlayson, B. L., \& McMahon, T. A. (2007). Updated world map of the Köppen-Geiger climate classification. Hydrology and earth system sciences, 11(5), 1633-1644.

Pereira, A. S., Shitsuka, D. M., Parreira, F. J. \& Shitsuka, R. (2018). Metodologia da pesquisa científica. UFSM. 119p. https://repositorio.ufsm.br/bitstream/handle/1/15824/Lic_Computacao_Metodologia-Pesquisa-Cientifica.pdf?sequence=1.

Pires, H. C. G., Rosa, L. D. S., Cabral, B. S., Silva, V. M. D., Nogueira, G. A., \& Ferreira, P. R. N. (2016). Padrão fenológico de Attalea maripa (Aubl.) Mart. em áreas de pastagens na Amazônia oriental. Floresta e Ambiente, 23(2), 170-179.

R Core Team (2020). R: A languageandenvironment for statisticalcomputing. R Foundation for StatisticalComputing, Vienna, Austria. URL https://www.Rproject.org/.

Satyanarayana, K. G., Guimarães, J. L., \&Wypych, F. E. R. N. A. N. D. O. (2007). Studiesonlignocellulosicfibers of Brazil. Part I: Source, production, morphology, properties and applications. CompositesPart A: AppliedScience and Manufacturing, 38(7), 1694-1709.

Souza, F., de Souza, E. H., de Padua, T. R. P., \& Ferreira, F. (2017). Abacaxizeiros (Ananas spp.) cultivados e silvestres. Embrapa Recursos Genéticos e Biotecnologia-Fôlder/Folheto/Cartilha (INFOTECA-E).

Tagliati, M. C. , Oliveira, H. C. , \& de Faria, A. P. G. (2018).Fenologia reprodutiva, recursos florais e polinização de espécies de bromeliaceae em um remanescente urbano de floresta atlântica do sudeste brasileiro Diversidade e Gestão 2(2): 139-150. 2018 Volume Especial: Conservação in situ e ex situ da Biodiversidade Brasileira e-ISSN: 2527-0044 139 .

Tropicos. Tropicos.org. Missouri Botanical Garden. 2014. http://www.tropicos.org .

Vogado, N. O., de Camargo, M. G. G., Locosselli, G. M., \& Morellato, L. P. C. (2016). Edge effects on the phenology of the guamirim, Myrcia guianensis (Myrtaceae), a cerrado tree, Brazil. Tropical Conservation Science, 9(1), 291-312. 